\title{
Universiteit
}

Leiden

The Netherlands

\section{Oxidation of Pd(553): From ultrahigh vacuum to atmospheric pressure} Westerström, R.; Gustafson, J.; Resta, A.; Mikkelsen, A.; Andersen, J.N.; Lundgren, E.; ... ; Stierle, A.

\section{Citation}

Westerström, R., Gustafson, J., Resta, A., Mikkelsen, A., Andersen, J. N., Lundgren, E., ... Stierle, A. (2007). Oxidation of Pd(553): From ultrahigh vacuum to atmospheric pressure. Physical Review B, 76, 155410. doi:10.1103/PhysRevB.76.155410

Version: $\quad$ Not Applicable (or Unknown)

License: $\quad$ Leiden University Non-exclusive license

Downloaded from: https://hdl.handle.net/1887/59985

Note: To cite this publication please use the final published version (if applicable). 


\title{
Oxidation of Pd(553): From ultrahigh vacuum to atmospheric pressure
}

\author{
R. Westerström, J. Gustafson, A. Resta, A. Mikkelsen, J. N. Andersen, and E. Lundgren \\ Department of Synchrotron Radiation Research, Lund University, Box 118, S-221 00 Lund, Sweden \\ N. Seriani and F. Mittendorfer \\ Institut für Materialphysik, Universität Wien, A-1090 Wien, Austria \\ M. Schmid, J. Klikovits, and P. Varga \\ Institut für Allgemeine Physik, Technische Universität Wien, A-1040 Wien, Austria \\ M. D. Ackermann and J. W. M. Frenken \\ Kamerlingh Onnes Laboratory, Leiden University, P.O. Box 9504, 2300 RA Leiden, The Netherlands \\ N. Kasper and A. Stierle \\ Max-Planck Institut für Metallforschung, Heisenbergstrasse 3, D-70569 Stuttgart, Germany
}

(Received 11 May 2007; published 11 October 2007)

\begin{abstract}
The oxidation of a vicinal $\operatorname{Pd}(553)$ surface has been studied from ultrahigh vacuum (UHV) to atmospheric oxygen pressures at elevated sample temperatures. The investigation combines traditional electron based UHV techniques such as high resolution core level spectroscopy, low-energy electron diffraction, scanning tunneling microscopy with in situ surface $\mathrm{x}$-ray diffraction, and ab initio simulations. In this way, we show that the $\mathrm{O}$ atoms preferentially adsorb at the step edges at oxygen pressures below $10^{-6}$ mbar and that (553) surface is preserved. In the pressure range between $10^{-6}$ and 1 mbar and at a sample temperature of $300-400{ }^{\circ} \mathrm{C}$, a surface oxide forms and rearranges the (553) surface facets and forming (332) facets. Most of the surface oxide can be described as a $\mathrm{PdO}(101)$ plane, similar to what has been found previously on other Pd surfaces. However, in the present case, the surface oxide is reconstructed along the step edges, and the stability of this structure is discussed. In addition, the $(\sqrt{6} \times \sqrt{6}) \mathrm{Pd}_{5} \mathrm{O}_{4}$ surface oxide can be observed on (111) terraces larger than those of the (332) terraces. Increasing the $\mathrm{O}$ pressure above 1 mbar results in the disappearance of the (332) facets and the formation of PdO bulk oxide.
\end{abstract}

DOI: 10.1103/PhysRevB.76.155410

\section{INTRODUCTION}

Most solid surfaces are exposed to oxygen in their natural environment, and they are often heavily influenced by the presence of this gas. Since in many processes of technological interest, such as catalysis, corrosion, and production of thin film devices, surface properties play a fundamental role, the understanding of oxide formation on solid surfaces is crucial.

In the last 40 years, interaction between oxygen and solid surfaces has been investigated by employing low-index single crystal surfaces in ultrahigh vacuum (UHV) and carefully depositing small amounts of atomic or molecular oxygen. This has led to detailed knowledge on adsorption sites, dissociation processes, and reaction pathways. However, the large differences between these simple UHV model systems and more real-world nonsingle crystal surfaces exposed to high pressures often prevent extrapolation of this knowledge to more realistic situations (materials and pressure gap ${ }^{1}$ ).

In recent years, methodological advances have made it possible to investigate single crystal surfaces of late transition metals exposed to high oxygen pressures with ex situ measurements, as well as with in situ oxidation, reactivity methods, and $a b$ initio simulations. ${ }^{2-22}$ These investigations have led to the discovery of well-ordered ultrathin surface oxides $^{5,12-20}$ at oxygen pressures up to 1 mbar and, at higher pressures, to the observation of bulk oxide formation. ${ }^{5,11}$ For-
PACS number(s): 68.35.Bs, 68.37.Ef, 68.47.Gh, 68.43.Bc

mation of oxide structures on the surface is expected to change dramatically its chemical properties; in fact, recent studies on the catalytic oxidation of $\mathrm{CO}$ have suggested that under realistic conditions, some of these oxide structures should be catalytically more active than the corresponding metal surfaces. ${ }^{2-4,12,23}$

In order to approach realistic conditions, the complexity of the model systems should also increase. From a morphological point of view, the well defined surfaces of low-index single crystals have little in common with the metal nanoparticles present in a real catalyst. A typical nanoparticle displays several different surface orientations, as well as a large number of defects, kinks, and steps. Such structural properties result in a much more complex oxidation behavior even under UHV conditions. ${ }^{24-26}$

A step toward higher complexity, but maintaining a well controlled surface, is the use of vicinal surfaces. ${ }^{27-32} \mathrm{~A}$ vicinal surface consists of a periodic array of steps, where the undercoordinated atoms at the step edges have similar properties as the atoms situated between different facets or at defects on a real metal particle. The reduced coordination of these step atoms can enhance the adsorption energy of the adsorbate, ${ }^{33,34}$ as well as promote the dissociation of molecules. ${ }^{35,36}$ Step sites can also enhance the reactivity despite a higher binding energy of one of the reactants at the step as compared to that on the terrace. ${ }^{32}$ 
In the present contribution, we have investigated the oxidation of a $\operatorname{Pd}(553)$ surface by using high resolution core level spectroscopy (HRCLS), scanning tunneling microscopy (STM), surface X-ray diffraction (SXRD), and ab initio simulations. We show that the $\operatorname{Pd}(553)$ surface undergoes large rearrangements already at low partial oxygen pressures. Such large rearrangements were also observed in our previous investigation of the oxidation of a $\mathrm{Rh}(553)$ surface. ${ }^{31}$ In the present case, the surface facets into (332) facets which are covered with a two-dimensional surface oxide at a pressure of $1 \times 10^{-5} \mathrm{mbar}$. This phase is stable up to $1 \mathrm{mbar}$ of oxygen, where the (332) facets disappear with the formation of PdO bulk oxide. From our studies, it is clear that a metal nanoparticle will change its shape together with other relevant properties such as the electronic structure governing the reactivity, already at a pressure above $1 \times 10^{-5}$ mbar. Such morphological and electronic changes of metal particles have been reported for the case of Ru particles. ${ }^{25} \mathrm{An}$ atomic understanding of these changes is of great importance, especially under real working conditions, for the comprehension of heterogeneous catalysis.

\section{EXPERIMENTAL AND COMPUTATIONAL SETUP}

The HRCLS measurements were done at room temperature at beamline $\mathrm{I} 311^{37}$ at MAX-lab, Lund, Sweden using a normal emission angle and photon energies of $400 \mathrm{eV}$ for the $\mathrm{Pd} 3 d_{5 / 2}$ level and $625 \mathrm{eV}$ for the $\mathrm{O} 1 s$.

The STM images were recorded in two different laboratories in Lund and Vienna, and the methods have been described in previous publications. ${ }^{5,14}$ Both STMs are positioned inside an ultrahigh vacuum system with a base pressure of $1 \times 10^{-10}$ mbar. STM tips used in these experiments were all electrochemically etched tungsten tips. All images shown were recorded in constant current mode.

The SXRD measurements were carried out at the MPI-MF beamline ${ }^{38}$ at Angströmquelle Karlsruhe (ANKA) using a photon energy of $10 \mathrm{keV}$ and at the ID3 surface diffraction beamline ${ }^{39}$ at the European Synchrotron Radiation Facility (ESRF) using a photon energy of $17 \mathrm{keV}$. The experimental end stations for these two beamlines are specifically constructed for studies under high pressures and elevated sample temperatures. ${ }^{40}$ The crystal basis used to describe the $(H K L)$ directions is a monoclinic basis set, which for the (553) coordinates gives $\mathbf{a}_{1}=\left(a_{0} / 2, a_{0} / 2,0\right), \mathbf{a}_{2}=($ $\left.-3 / 2 a_{0}, 0,5 / 2 a_{0}\right)$, and $\mathbf{a}_{3}=\left(5 a_{0},-5 a_{0}, 3 a_{0}\right)$, expressed as a function of the cubic Pd lattice, with $\mathbf{a}_{1}$ and $\mathbf{a}_{2}$ lying in the surface plane with lengths of 2.751 and $11.34 \AA$ and $\mathbf{a}_{3}$ out of plane with length of $29.88 \AA$. In this basis, $\alpha=\beta=90^{\circ}$ and $\gamma=111.3^{\circ}\left(a_{0}=3.89 \AA\right.$ is the bulk Pd lattice constant $)$.

The calculations were performed using the Vienna $a b$ initio simulation package (VASP), ${ }^{41,42}$ using PAW potentials, ${ }^{43,44}$ the PW91 exchange-correlation functional, ${ }^{45}$ and a cutoff energy of $250 \mathrm{eV}$. The Pd surface has been modeled by a periodically repeated slab consisting of six layers parallel to the (111) terrace with the density functional theory-generalized gradient approximation (DFT-GGA) lattice constant of $3.95 \AA$. The core level shifts (CLSs) have been calculated including final state contributions.

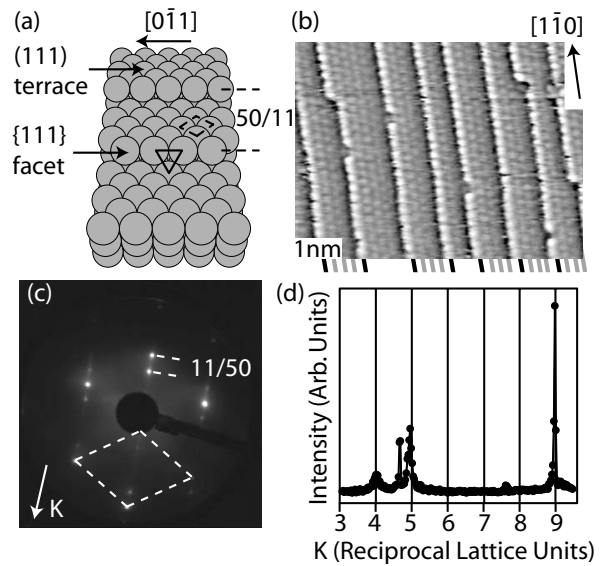

FIG. 1. (a) Model of the Pd(553) surface. (b) STM image of the clean surface $\left(85 \times 65 \AA^{2}, 0.015 \mathrm{~V}\right.$, and $\left.0.54 \mathrm{nA}\right)$. Almost all terraces consist of one step row and three visible rows in the terrace that together with a fifth row underneath the next step make up the (553) surface structure. (c) LEED pattern from the clean $\operatorname{Pd}(553)$ surface. The unit cell of the (111) terraces is indicated and the splitting due to the periodicity of the steps. (d) SXRD scan from the $\operatorname{Pd}(553)$ surface in the $K$ direction with $H=0$ and $L=1.2$ plotted in (553) coordinates. The extra peak between $H=4$ and $H=5$ is due to $\{111\}$ planes from polycrystalline parts within the sample.

\section{RESULTS AND DISCUSSION}

\section{A. Clean surface}

Figure 1(a) shows a model of the $\operatorname{Pd}(553)$ surface, which consists of (111) terraces that are five atom rows wide and separated by monatomic $\{111\}$ faceted steps $10.56 \AA$ apart. Comparing the atomically resolved STM image in Fig. 1(b) and the model confirms that, with the exception of some small variations in terrace width and other imperfections such as vacancies and kinks, the surface has the expected structure. The presence of a well-ordered (553) surface is further supported by low-energy electron diffraction (LEED) and SXRD measurements, see Figs. 1(c) and 1(d). Inspecting the LEED pattern, the step structure appears as a splitting of the (111) spots, which corresponds to the terrace width of the (553) surface. In the SXRD scan, diffraction peaks appear at integer values of $K$ when plotted in (553) coordinates.

\section{B. Overview}

In this section, we describe the general development of the oxidation of the $\operatorname{Pd}(553)$ surface as observed with HRCLS and SXRD. Figure 2(a) shows HRCLS from the O $1 s$ and the $\mathrm{Pd} 3 d_{5 / 2}$ regions as the oxygen exposure increases. In this case, the measurements are performed ex situ after the surface has been exposed for $300 \mathrm{~s}$ at the indicated pressure and a sample temperature of $350{ }^{\circ} \mathrm{C}$. Starting with the clean surface, the $\mathrm{Pd} 3 d_{5 / 2}$ does not exhibit well-separated surface and step components but rather resembles the HRCLS of the $\operatorname{Pd}(111)$ surface. ${ }^{13}$ As the $\mathrm{O}$ coverage increases, a shoulder appears in the $\mathrm{Pd} 3 d_{5 / 2}$ level toward higher binding energy at a pressure of $10^{-6}$ mbar. Such a shift is consistent with surface or step-edge Pd atoms coordinated to one or two $\mathrm{O}$ atoms. ${ }^{14}$ This shoulder persists until a pressure of $10^{-5} \mathrm{mbar}$, 
(a)

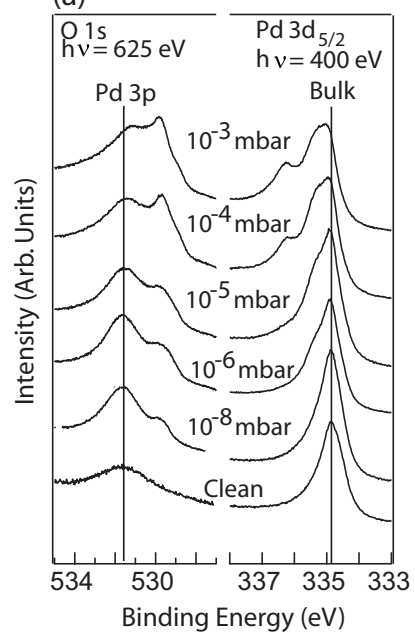

(b)

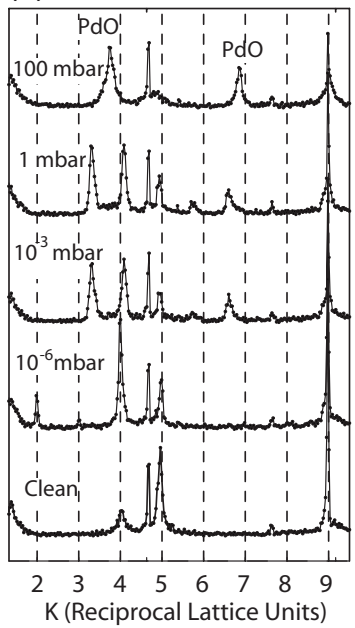

FIG. 2. (a) Ex situ HRCLS spectra for different oxygen exposures. Each spectrum was recorded at room temperature after exposing the sample to oxygen for $5 \mathrm{~min}$ at $350{ }^{\circ} \mathrm{C}$. (b) In situ SXRD scans in the $k$ direction with $H=0$ and $L=1.2$ for different oxygen pressures and a sample temperature of $350{ }^{\circ} \mathrm{C}$. The scans are plotted in (553) coordinates.

at which an additional component at higher binding energies appears, which increases in intensity until the final pressure of $10^{-3}$ mbar. In addition, the intensity of the $\mathrm{Pd} 3 d_{5 / 2}$ component corresponding to $\mathrm{Pd}$ atoms coordinated to one or two $\mathrm{O}$ atoms increases simultaneously with the growth of the new component. Turning to the $\mathrm{O} 1 s$ region, it should be noted that the Pd $3 p$ level has a binding energy close to the $\mathrm{O} 1 s$ level. As the O pressure is increased, one single $\mathrm{O} 1 s$ component can be observed, which increases its intensity as the oxygen exposure increases. Furthermore, a shift in conjunction with a broadening is observed as the pressure reaches $10^{-6}$ mbar. Above this pressure, an additional shoulder appears in the $\mathrm{O} 1 \mathrm{~s}$ spectrum, indicating the existence of two distinctly different $\mathrm{O}$ atoms at the surface and the formation of a surface oxide. ${ }^{13,14}$

The SXRD measurements were performed by doing $K$ scans for $H=0$ and $L=1.2$, as shown in Fig. 2(b). The signature of the clean surface can be followed in situ to a pressure of $10^{-7}$ mbar. Up to this pressure, diffracted intensities at the integer-order positions corresponding to the clean $\operatorname{Pd}(553)$ surface can be detected. No particular changes in the diffraction indicating large surface rearrangements can be observed at these pressures. At $10^{-6}$ mbar, the intensity of some of the peaks, in particular, the intensity at $K=2$, changes significantly. This indicates that the local atomic arrangement at the $\operatorname{Pd}(553)$ has changed, however, without disrupting the (553) surface. The behavior will be discussed in more detail below.

Increasing the pressure above $10^{-6} \mathrm{mbar}$, new peaks appear at noninteger values, clearly demonstrating a large rearrangement of the surface. Again, this new surface phase will be discussed below, which involves surface faceting and the formation of a surface oxide. These new peaks are stable up to a pressure of 1 mbar. Above 1 mbar, additional new peaks can be seen in the $K$ scans, and we will show below that these particular peaks correspond to the formation of $\mathrm{PdO}$.
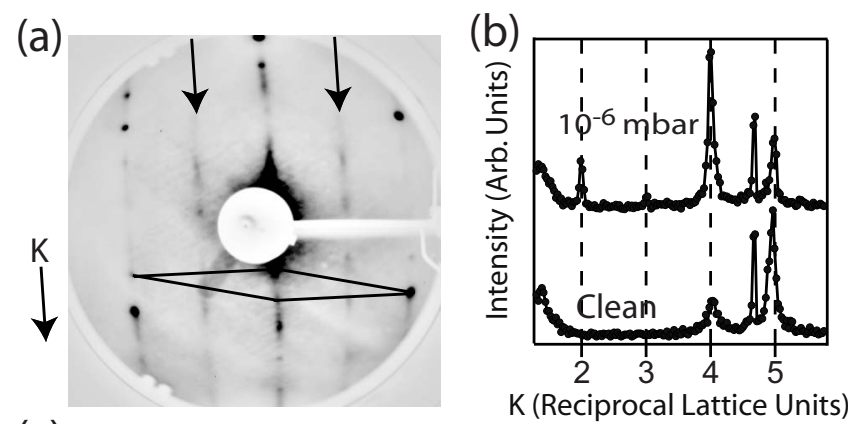

(c)

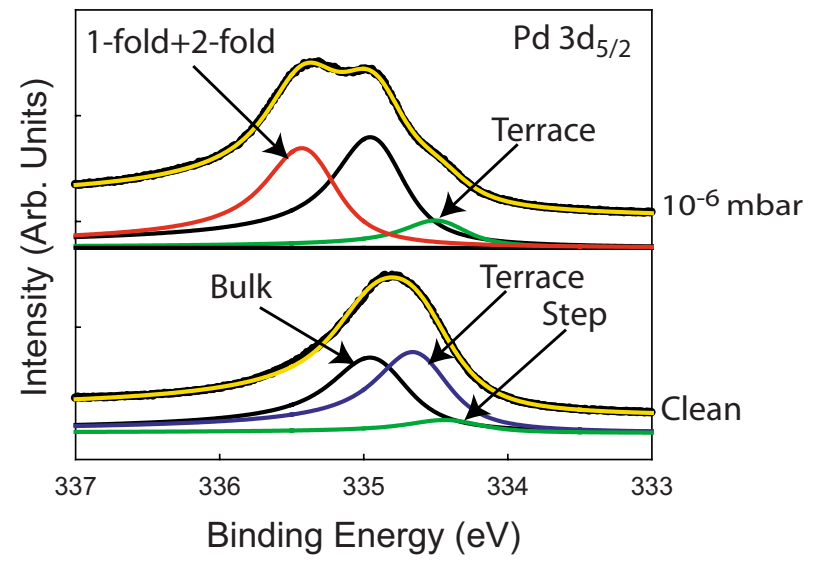

FIG. 3. (Color online) (a) LEED image from the $\operatorname{Pd}(553)$ surface after exposing it to $10^{-6} \mathrm{mbar}$ of oxygen for $5 \mathrm{~min}$ at $350{ }^{\circ} \mathrm{C}$. Apart from the spots from the clean surface, also $(2 \times 1)$-like streaks can be seen, indicating a structure with a periodicity that is well defined in the step direction. (b) In situ SXRD $K$ scans $(H$ $=0$ and $L=1.2$ ) from the clean and the $10^{-6}$ mbar oxygen exposed $\operatorname{Pd}(553)$ surface. (c) HRCLS from the Pd $3 d_{5 / 2}$ level from the clean (bottom) and the $10^{-6}$ mbar oxygen exposed surface (top) for $5 \mathrm{~min}$ at $350{ }^{\circ} \mathrm{C}$.

\section{Low coverage (clean to $\mathbf{1 0}^{-6}$ mbar)}

Figure 3(a) shows a LEED image after exposing the crystal to $10^{-6}$ mbar of oxygen for $5 \mathrm{~min}$ at a temperature of $350{ }^{\circ} \mathrm{C}$. In addition to the spots from the clean (553) surface, $(2 \times 1)$-like streaks can be observed. This indicates a structure with a well defined periodicity in the $[01 \overline{1}]$ direction but less ordered across the steps.

The fact that the surface structure is modified at these conditions is confirmed by the SXRD results shown in Fig. 3(b). Here, we observe a change of the intensity of the integer-order diffraction. This change is already a possible indication of strong relaxation effects of the surface, in particular, of step-edge atoms.

Turning to the HRCL measurements, the bottom spectrum in Fig. 3 shows the $\mathrm{Pd} 3 d_{5 / 2}$ core level region from the clean $\operatorname{Pd}(553)$ surface. The spectrum can be decomposed into three different components corresponding to emission from the bulk, the terrace atoms (named T1-T4 in Table I), and the step atoms (step). A comparison between measured and calculated core level binding energy shifts is shown in Table I and the agreement can be seen to be satisfactory.

Exposing the surface to $10^{-6}$ mbar of oxygen results in the upper HRCL spectra in Fig. 3(c). In this case, the decom- 
TABLE I. Core level shifts for the Pd $3 d$ states from the clean $\operatorname{Pd}(553)$ surface atoms: calculated and experimental core level shifts. A Pd atom in the fourth metallic layer was taken as bulk reference for the calculations.

\begin{tabular}{lcc}
\hline \hline & $\begin{array}{c}\text { Calculated CLS } \\
(\mathrm{eV})\end{array}$ & $\begin{array}{c}\text { Experimental CLS } \\
(\mathrm{eV})\end{array}$ \\
\hline $\mathrm{Pd}_{\text {step }}$ & -0.45 & -0.52 \\
$\mathrm{Pd}_{\mathrm{T} 1}$ & -0.25 & -0.29 \\
$\mathrm{Pd}_{\mathrm{T} 2}$ & -0.20 & -0.29 \\
$\mathrm{Pd}_{\mathrm{T} 3}$ & -0.25 & -0.29 \\
$\mathrm{Pd}_{\mathrm{T} 4}$ & -0.05 & \\
\hline \hline
\end{tabular}

position yields three components from the $\mathrm{Pd} 3 d_{5 / 2}$ spectra. The first originates from the bulk Pd atoms, and the second from $\mathrm{Pd}$ atoms at the surface displaying a surface core level shift of approximately $-0.44 \mathrm{eV}$, revealing that some of the atoms at the terraces are not coordinated to oxygen atoms. The third component can be found at higher binding energy, which relates to $\mathrm{O}$ atoms adsorbed on the steps and/or at the terrace.

The DFT calculations give an insight on atomistic changes upon oxygen adsorption. In the low-coverage case, oxygen tends to accumulate in the vicinity of the upper step edge [Fig. 4(a)]. The adsorption energy is significantly increased to a value of $E_{a d s}=-1.76 \mathrm{eV}$ as compared to a $p(2$ $\times 2)-\mathrm{O}$ on the $(111)$ terrace $(-1.36 \mathrm{eV})$. In contrast to the adsorption of oxygen on stepped $\mathrm{Rh}$ surfaces, ${ }^{31}$ the driving force is large enough to favor a further occupation of the step before oxygen adsorption on the terrace, leading to a p2mg-like zigzag pattern at the step [Fig. 4(b)]. If the oxygen partial pressure is increased even further, additional oxygen (a)

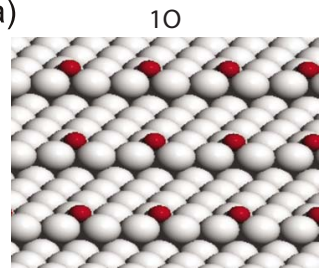

(c)

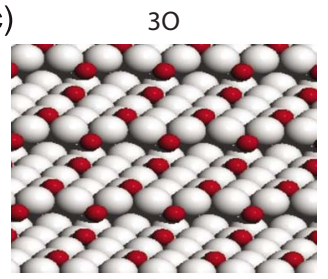

(b)

(d)
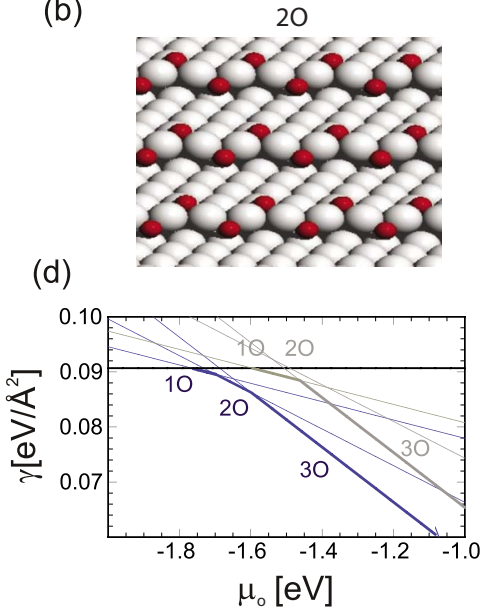

FIG. 4. (Color online) Calculated models for the adsorption of oxygen on $\operatorname{Pd}(553)$ : (a) the low-coverage case (1O), (b) a $p 2 m g$-like structure at the step (2O), (c) oxygen adsorbed on the step edge and terrace (3O), and (d) phase diagram of the relaxed phases [(a)-(c)] (lower blue lines) as well as the corresponding unrelaxed structures (light gray lines). $\mu_{0}$ is the chemical potential of oxygen and $\gamma$ the Gibbs free surface energy. High chemical potentials correspond to high oxygen pressure and/or low temperature.
TABLE II. Calculated and experimental core level shifts for the $\operatorname{Pd} 3 d$ states of the Pd surface atoms in the $\mathrm{Pd}(553) 3 \mathrm{O}$ adsorption structure as shown in Fig. 4(c). For the atoms in the terrace (T1 is closest to the step), the two different types coordinated to one and zero oxygen atoms are named "10" and "no O," respectively. A Pd atom in the fourth metallic layer was taken as bulk reference for the calculations.

\begin{tabular}{lcc}
\hline \hline & $\begin{array}{c}\text { Calculated CLS } \\
(\mathrm{eV})\end{array}$ & $\begin{array}{c}\text { Experimental CLS } \\
(\mathrm{eV})\end{array}$ \\
\hline $\mathrm{Pd}_{\text {step-2O }}$ & +0.32 & +0.47 \\
$\mathrm{Pd}_{\mathrm{T} 1-1 \mathrm{O}}$ & +0.35 & +0.47 \\
$\mathrm{Pd}_{\mathrm{T} 1-\text { no O }}$ & -0.53 & -0.44 \\
$\mathrm{Pd}_{\mathrm{T} 2-1 \mathrm{O}}$ & +0.32 & +0.47 \\
$\mathrm{Pd}_{\mathrm{T} 3-1 \mathrm{O}}$ & +0.28 & +0.47 \\
$\mathrm{Pd}_{\mathrm{T} 3-\text { no O }}$ & -0.49 & -0.44 \\
\hline \hline
\end{tabular}

is adsorbed at the terrace [Fig. 4(c)]. This phase is thermodynamically stable over a wide pressure range [Fig. 4(d)]. The phase diagram for the relaxed $1 \mathrm{O}, 2 \mathrm{O}$, and $3 \mathrm{O}$ is shown in [Fig. 4(d)] with the lower (blue) line. The adsorption of oxygen leads to an expansion of the lattice perpendicular to the step. For the $p 2 m g$-like structure, the nearest-neighbor distances of the Pd atoms with oxygen bonds are expanded up to a value of $2.99 \AA$, an increase of $0.19 \AA$ compared to the calculated GGA bulk distance. In Fig. 4(d), the phase diagram for the corresponding unrelaxed structures is also shown (light gray lines). Interestingly, comparison shows that the stability of the $2 \mathrm{O}$ and $3 \mathrm{O}$ structure is significantly enhanced due to the relaxation effects.

The calculated Pd $3 d$ core level shifts shown in Table II reveal two opposed tendencies: the core level states of the Pd atoms sharing one or two oxygen bonds are shifted to higher energies with a common shift of $0.3 \mathrm{eV}$ with respect to bulk Pd. On the other hand, the states of the remaining atoms without coordination to $\mathrm{O}$ atoms are shifted to lower energies by $-0.5 \mathrm{eV}$. Again, we find that the overall agreement between calculated and experimental values is good.

In summary, we find that at low oxygen pressures $\left(10^{-6}\right.$ mbar range $)$, the $\mathrm{O}$ atoms adsorb in a zigzag fashion along the step edge as well as in a one-dimensional $(2 \times 1)$ structure on the terraces. Strong relaxations in the Pd surface atoms are observed, already indicating potential major rearrangements of the surface at higher $\mathrm{O}$ pressure; however, at this low $\mathrm{O}$ pressure, no strong faceting or surface reconstruction occurs.

\section{Higher coverage $\left(10^{-5}-1\right.$ mbar $)$ : Faceting and formation of a surface oxide}

As the oxygen pressure is increased above $10^{-5}$ mbar, a change in the surface structure can be seen in the LEED image, see Fig. 5(a). The spots from the (111) terraces are still present but the spot-spot distance of the splitting has decreased to a value below that of a (553) surface, indicating a longer periodicity across the steps. Perpendicular to the $K$ direction, there are also additional spots with a periodicity of 

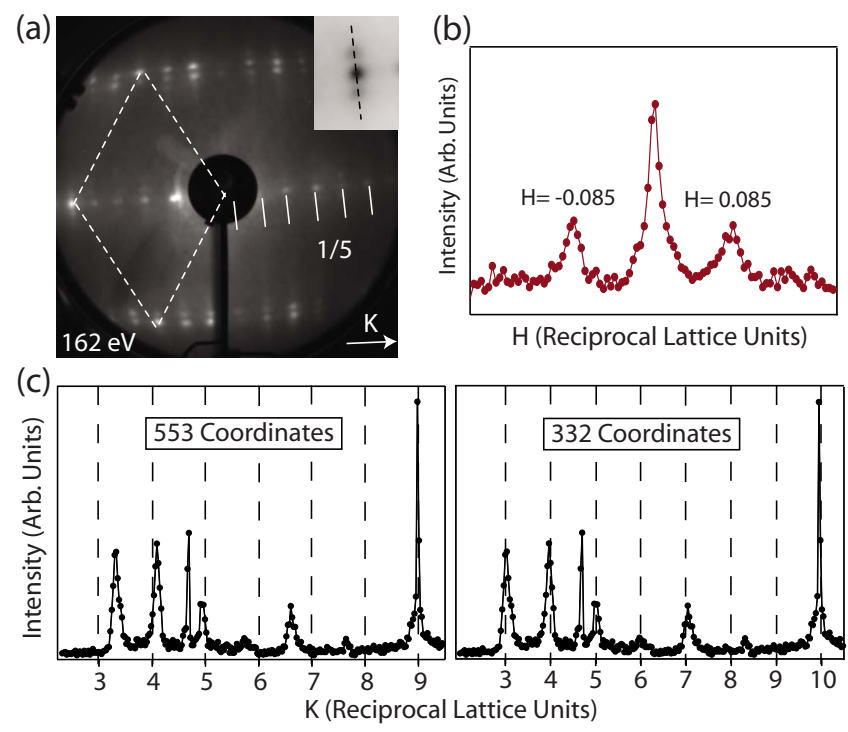

FIG. 5. (Color online) (a) LEED image after exposing the sample to $10^{-3}$ mbar of oxygen at $350{ }^{\circ} \mathrm{C}$. The periodicity of the spots in the $K$ direction has changed due to faceting. New spots have emerged perpendicular to the steps, indicating a reconstruction along the steps. (b) SXRD scan along the steps reveals a periodicity of about 12. (c) SXRD scans along $K$ with $H=0$ and increasing $L$, plotted both in (553) and (332) coordinates, showing that the (553) surface has faceted into a (332) surface.

approximately 12. From this LEED pattern, we can conclude that the surface has faceted, resulting in wider terraces, and reconstructed parallel to the steps. By using SXRD, we can make a more precise determination of the surface structure. A facet on a surface will give rise to a crystal truncation rod
(CTR) normal to its surface plane. A facet with a different orientation will have CTRs that are tilted with respect to the CTRs from the (553) surface, as well as having a different periodicity in the plane. As a consequence of this, when plotted in $\operatorname{Pd}(553)$ coordinates, the intensity peaks from different facets will not appear at integer values of $K$. Figure 5(c) shows a $K$ scan (in-plane scan perpendicular to the step edges) and $H=0$. The peaks that were characteristic for the (553) surface have disappeared, and instead new peaks appear at noninteger values of $K$. This shows that the surface structure has changed so that the average periodicity differs from that of the (553) surface. The LEED image in Fig. 5(a) indicated that the step width had increased to $12.9 \AA$, which corresponds to the step distance of a (332) surface. If we now plot the same $K$ scan in the (332) coordinates, the peaks appear at integer values, yielding strong support to the idea that the average periodicity is that of a (332) surface [Fig. $5(\mathrm{c})]$

Along the step edges (perpendicular to the $K$ direction), the LEED image [Fig. 5(a)] displayed additional spots, originating from a new structure. A SXRD scan in this direction also reveals a reconstruction along the steps with a periodicity of about 12, see Fig. 5(b). The (332) facets and the accompanying surface reconstruction along the step edges are stable up to a pressure of $1 \mathrm{mbar}$, as probed by in situ SXRD.

The STM image in Fig. 6(a) of the Pd(553) surface after $10^{-3}$ mbar of oxygen at $350{ }^{\circ} \mathrm{C}$, agrees with the observation from the diffraction studies. (553) terraces have almost completely disappeared. Instead, the surface consists of larger areas, each of which accommodates up to six terraces with a periodicity of approximately $13 \AA$, corresponding to that of the (332) surface, as shown in the line scan in Fig. 6(b).
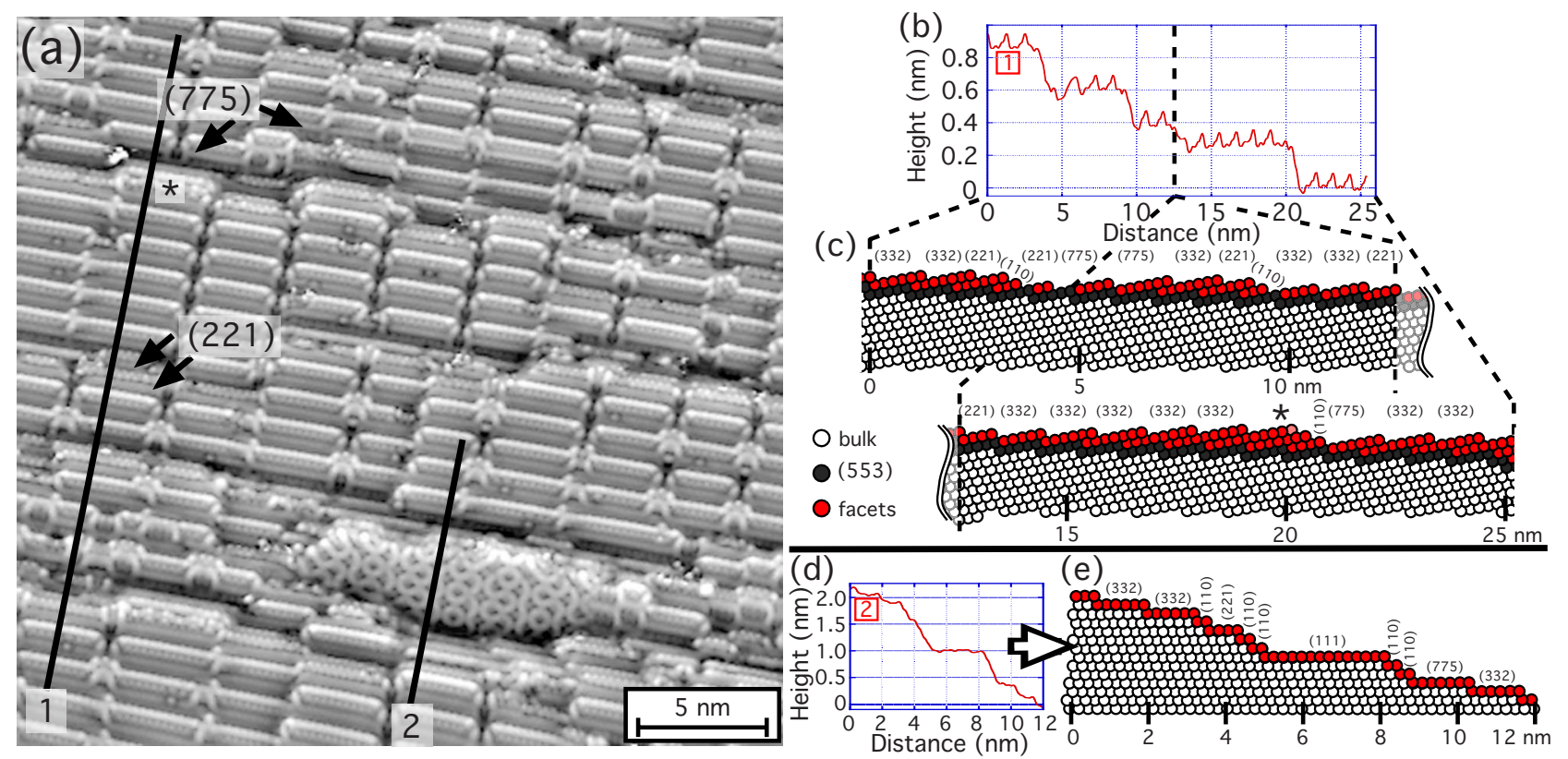

FIG. 6. (Color online) (a) STM image $(V=0.95 \mathrm{~V}, I=0.64 \mathrm{nA})$ after exposure to $10^{-3}$ mbar of oxygen at $350{ }^{\circ} \mathrm{C}$. On the (221) and (775) areas (marked by arrows), a similar surface oxide as on (332) can be observed. (b) and (d) are line scans, which are marked by black lines in (a) and have been tilted to obtain flat (332) and (111) facets, respectively. (c) and (e) are the corresponding side view models. The asterisk in (a) and in model (c) marks a disordered area of the STM image. 

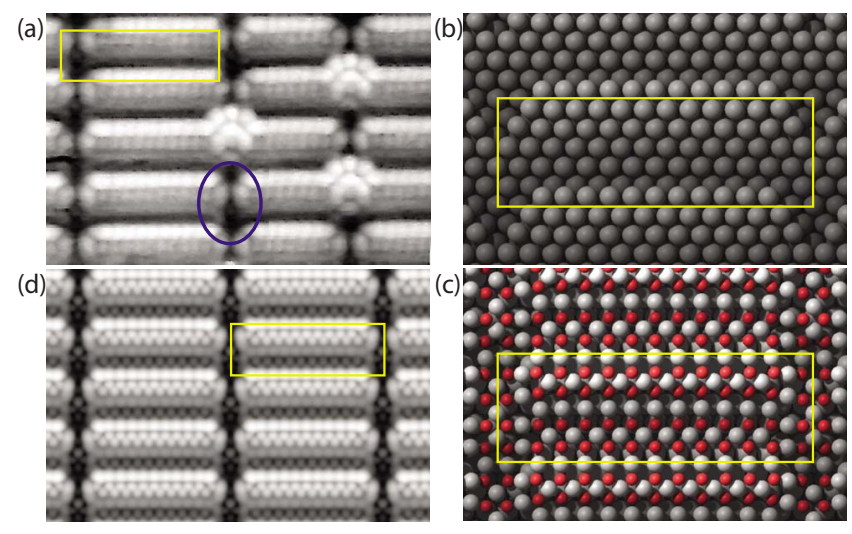

FIG. 7. (Color online) (a) STM image $(V=0.21 \mathrm{~V}, I=5.69 \mathrm{nA})$ of an oxide-covered (332) area after exposure to $10^{-3}$ mbar of oxygen at $350{ }^{\circ} \mathrm{C}$. The oval indicates the transition region between well-ordered surface-oxide stripes. The oxide unit cell is indicated by a rectangle in (a)-(d). (b) Model of the substrate below the surface oxide and (c) simulated lowest-energy structure for the surface oxide on $\mathrm{Pd}(332)$. The red (small) atoms are oxygen, while gray (large) atoms are $\mathrm{Pd}$, and the geometric height (normal to the surface) is indicated by atom brightness. (d) Simulated STM image of the lowest-energy structure on $\operatorname{Pd}(332)$. States with energy between $E_{F}$ and $E_{F}+1 \mathrm{eV}$ have been taken into account.

Thus, we conclude that the majority of the surface has faceted into (332). However, as it can be seen in the structure model in Fig. 6(c), terraces of different widths can be found between the extended (332) areas. Besides (332), the facets in the line profile include (110), (221), and (775). The structure model [Fig. 6(c)] explains the line profile of Fig. 6(b) excellently and also demonstrates how an overall (553) surface orientation is preserved by introducing steeper facets, such as (110). Thus, the average slope of the line scan in Fig. 6(b) runs only $0.2^{\circ}$ off a (553) surface orientation.

In the lower part of the STM image, a (111) terrace can be found. This area is covered by the " $\sqrt{6}$ " surface oxide, identified by its characteristic "asian-carpet-like" pattern. ${ }^{13}$ As indicated by the line scan in Fig. 6(d) and its structure model in Fig. 6(e), terrace widths in direct proximity of the (111) area almost exclusively correspond to a (110) orientation. Due to this step bunching, an overall (553) surface orientation is again preserved.

A structural model for the (332) surface oxide can be constructed from the STM images [see Figs. 6(a) and 7(a)]. The distance between the bright spots along the steps on the (332) terraces is slightly less than $3 \AA$, as determined from STM, corresponding roughly to the short side of the PdO bulk unit cell $(3.04 \AA$, cf. Sec. III E). Also, the $(\sqrt{5} \times \sqrt{5})$ surface oxide on $\mathrm{Pd}(100)$ consists of $\mathrm{PdO}(101)$ planes with the dimension $3.04 \times 6.15 \AA^{2} .{ }^{14,20}$ The mismatch between the (332) step distance $(12.9 \AA)$ and two long sides of the $\mathrm{PdO}(101)$ cell is only $0.6 \AA$. Thus, there is a strong indication that the structure on the (332) terraces consists of PdOtype building blocks, in a similar way as in the case of $\mathrm{Pd}(100) .{ }^{14,20}$ The use of PdO building blocks for the new structure is also supported by its appearance in STM, which is similar to the surface oxide on $\operatorname{Pd}(100)$, consisting of these

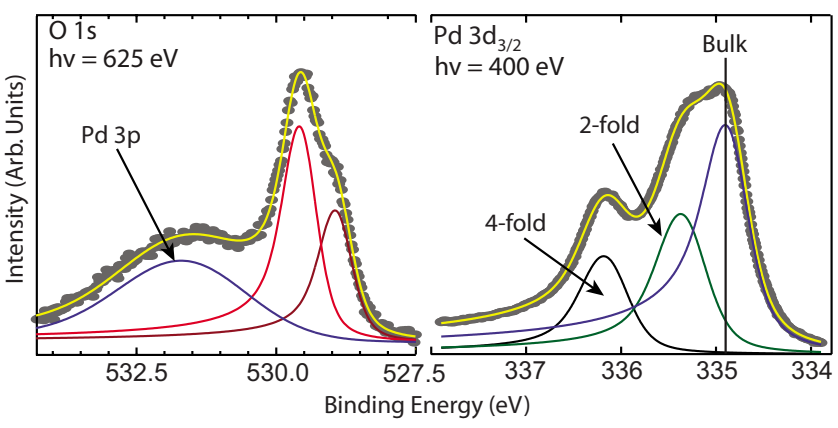

FIG. 8. (Color online) HRCLS from the surface oxide on $\mathrm{Pd}(553)$ : left the $\mathrm{O} 1 s$ region, and right the $\mathrm{Pd} 3 d_{5 / 2}$ region.

building blocks. ${ }^{14,20}$ Bright stripes on the (332) terraces running parallel to the steps in Fig. 6(a) can be described by alternating rows of twofold and fourfold oxygen-coordinated $\mathrm{Pd}$. The bright row directly at the step edge can be attributed to twofold Pd, followed by a row of fourfold and another row of twofold $\mathrm{Pd}$. A second fourfold row at the rear of the (332) terrace is invisible in the STM measurement.

Furthermore, along the steps, a reconstruction with a periodicity of 10-16 Pd distances is found by STM, in agreement with the observations by LEED and SXRD as presented above. The $\mathrm{PdO}(101)$ planes on the (332) terraces are interrupted with this periodicity. Without interruption, the misfit of the atomic distances along the steps within the surface oxide layer $(\leqslant 3 \AA)$ and the substrate $(2.75 \AA)$ would force some atoms into unfavorable sites. Experimentally, several different structures in the transition areas can be observed [see Fig. 6(a)], of which the most common transition structure is indicated by an oval in Fig. 7(a). Several different models for this transition structure have been investigated. The one with the lowest energy in DFT calculations is shown in Fig. 7(c). Its transition areas are strongly reconstructed, including Pd vacancies in the layer below the surface oxide [see Fig. 7(b)]. Nevertheless, the surface oxide structure in the transition area is still related to the uninterrupted oxide. In principle, a PdO-like structure, which is rotated by $90^{\circ}$ and matches the (332) terrace width of $12.9 \AA$, forms the transition, having only twofold and fourfold oxygen-coordinated $\mathrm{Pd}$ atoms. The unit cell in Fig. 7(c) contains 32 twofold and 23 fourfold coordinated Pd atoms. A calculated STM image for this lowest-energy model is shown in Fig. 7(d). The agreement with the measurement is excellent since the model is capable of not only reproducing the correct brightness contrast in the main structure on the (332) terraces but also in the transition area, confirming the formation of a $\mathrm{PdO}(101)$-like surface oxide on the (332) faceted areas.

Figure 8 shows the HRCLS results from the (332) faceted surface. The $\operatorname{Pd} 3 d_{5 / 2}$ spectrum consists of a bulk peak and two additional components. The component with the highest binding energy comes from $\mathrm{Pd}$ atoms that are coordinated to four oxygen atoms, while the middle peak can be assigned to $\mathrm{Pd}$ atoms with two neighboring oxygen atoms. ${ }^{13,14,20}$ The two-fold/four-fold ratio is found to be $4: 3$, in excellent agreement with the model shown in Fig. 7(c) with a ratio of 32:23.

In the $\mathrm{O} 1 \mathrm{~s}$ peak, two components can be detected, the ratio between the two components being $2: 3$. The simulation 
shows a more complex picture. Each of the four oxygen rows in Fig. 7(c) has a different CLS. In the second row from the upper edge of the unit cell in Fig. 7(c), the $1 s$ states are more bound by $0.3 \mathrm{eV}$ in comparison with the first row above the step, in the third by $0.5 \mathrm{eV}$, and in the fourth by $0.4 \mathrm{eV}$. Moreover, the oxygens near the transition region still have different CLSs with respect to their row [typically similar to those in the first row in Fig. 7(c)], leading presumably to a rather complex spectrum. Still, some of the peaks are very close to each other, which explains why using only two components to fit the experimental data is successful (see Fig. 8).

As mentioned previously, the matching of two cells of a $\mathrm{PdO}(101)$ layer with the step-step distance of $\mathrm{Pd}(332)$ and the lack of such a matching for (553) terraces explain the formation of (332) facets by oxidation. Furthermore, the misfit of the oxide layer (interatomic distances $2.84 \AA$ according to DFT) and the substrate $(2.75 \AA)$ along the steps causes the formation of a periodically occurring transition structure. In this transition structure, oxidation causes Pd vacancies in the topmost substrate layer directly below the surface oxide, resulting in the formation of kinks, which seem to provide favorable sites for oxide layer atoms. Thus, we observed that a carpetlike oxide layer completely covers the stepped (332)faceted areas.

As already mentioned, introducing different facets, most of which are steeper than (553), ensures that the requirement of an overall (553) surface orientation can be fulfilled. In Fig. 6(a), a surface oxide is clearly visible on the (775) and (221) facets. The (775) area (marked by arrows) clearly displays an oxide structure similar to that on (332), with one more row of $\mathrm{Pd}$, due to the increased terrace width. On the other hand, the oxide on the (221) facet (arrows) appears similar to the oxide on (332) facets at the rear part of the terrace but is prematurely interrupted due to the reduced terrace width. Therefore, it is very likely that a similar oxide carpet as on extended (332) areas covers all other facets as well, ranging from steep (110) to flat (775) facets. One can thus argue that the sequence of facets is self-organized in such a way that it provides a favorable substrate for the carpetlike oxide layer.

It is noticeable that the (111) terraces of $\operatorname{Pd}(332)$ facets do not display a surface oxide that is structurally similar to the $\sqrt{6}$ surface oxide on a regular $\operatorname{Pd}(111)$ surface, ${ }^{13}$ but rather to that on $\operatorname{Pd}(100),{ }^{14,20}$ with part of the terrace covered by a transition structure different from both. Nevertheless, the basic building principle of having a single oxide layer, consisting of a regular sequence of twofold and fourfold oxygencoordinated Pd, can be found in all these structures. This can explain the rather similar stability for the $\sqrt{6}$ structure on $\operatorname{Pd}(111)$ and the surface oxide on $\operatorname{Pd}(332)$ (see Fig. 9). Here, we compare the stability of the present surface oxide with the

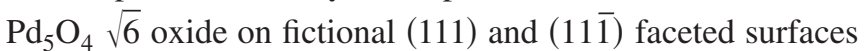
and we find that the $\operatorname{Pd}\{111\}-\sqrt{6}$ structure is marginally more stable at the experimental conditions of interest $\left(\mu_{\mathrm{O}}\right.$ $\approx 1 \mathrm{eV}$ ), but the difference is very small. Furthermore, since faceting of $\operatorname{Pd}(553)$ is not limited to (332) facets-but to a rather broad spectrum of different terrace widths-and since step bunching into (110) in direct proximity of (111) facets occurs [see Figs. 6(d) and 6(e)], a combination of $\operatorname{Pd}(111)$

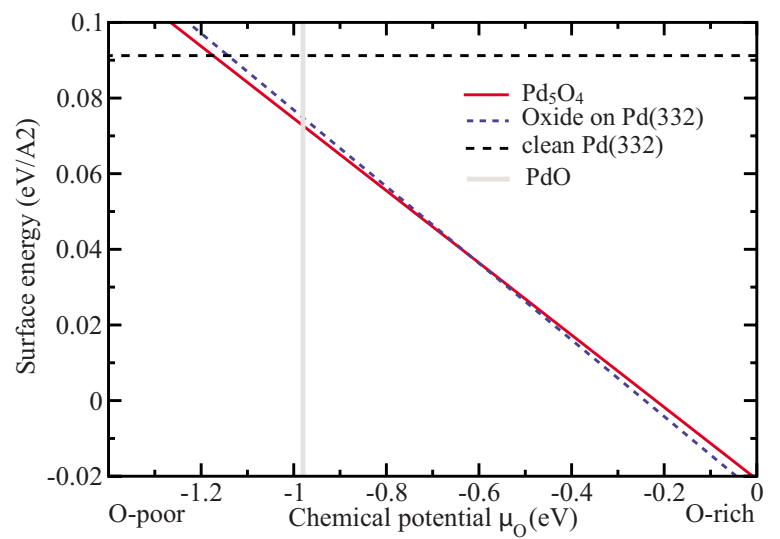

FIG. 9. (Color online) Phase diagram for the $\mathrm{Pd}_{5} \mathrm{O}_{4}$ surface oxide (" $\sqrt{6}$ ") on $\mathrm{Pd}(111)$ as well as the $\mathrm{PdO}(101)$-like surface oxide layer on Pd(332), as shown in Fig. 7(c). The surface energy for the $\sqrt{6}$ oxide is multiplied by 1.1078 to account for the higher surface area that fictional (111) and (11 $\overline{1})$ faceted surfaces would result in. The vertical gray line corresponds to the stability limit of the $\mathrm{PdO}$ bulk oxide.

facets covered by the $\sqrt{6}$ phase and other surface-oxidecovered facets such as $\operatorname{Pd}(110)$ might be slightly more stable than $\operatorname{Pd}(111)+\operatorname{Pd}(11 \overline{1})$. Therefore, the phase diagram (Fig. 9) does not necessarily contain the thermodynamically stable configuration. The reason why the (332) oxide is so common compared to the $\sqrt{6}$ oxide on extended (111) areas could also be due to kinetics. The formation of (332) facets from a (553) surface requires fewer atomic rearrangements and (332) facets already provide a step-step distance well suited for accommodating a surface oxide. Surface oxide formation on (332) facets might then hinder the formation of larger (111) terraces and thus the growth of the $\sqrt{6}$ surface oxide to such an extent that the bulk PdO oxide (cf. Sec. III E) is formed prior to complete transformation into (111) facets with the $\sqrt{6}$ oxide.

\section{E. High pressure (1-100 mbar): The formation of PdO}

The SXRD scans in Fig. 2(b) show that the (332) facets are stable up to 1 mbar of oxygen and thus also the $\mathrm{PdO}(101)$ surface oxide. By looking closely at the $K$ scan recorded at an oxygen pressure of 1 mbar, a new diffraction peak appearing between $K=3$ and $K=4$ marked $\mathrm{PdO}$ can be seen. Increasing the pressure to $10 \mathrm{mbar}$ results in the disappearance of the diffraction from both the (332) and the (553) facets at this $L$ value. Instead, the peak between $K=3$ and $K=4$ continues to grow in conjunction with a second peak located between $K=6$ and $K=7$. These two new peaks can be assigned to the bulk oxide PdO. ${ }^{46-48}$ Figure 10 presents the intensity distribution in the $H=0$ plane. The Bragg peaks of PdO can be either (002) and (004) or (101) and (202). Additional scans (not shown) along the step edges reveal a periodicity corresponding to the side $a$ of the PdO unit cell $(3.04 \AA)$. The angle between the direction of the $\mathrm{PdO}$ peaks and the $\operatorname{Pd}(553)$ surface is roughly $16^{\circ}$ (the broken line in Fig. 10). This is very close to the angle of $15.9^{\circ}$ between the 
(a)

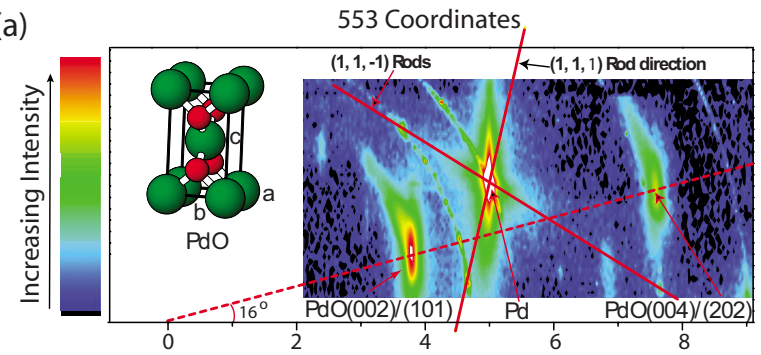

(b)

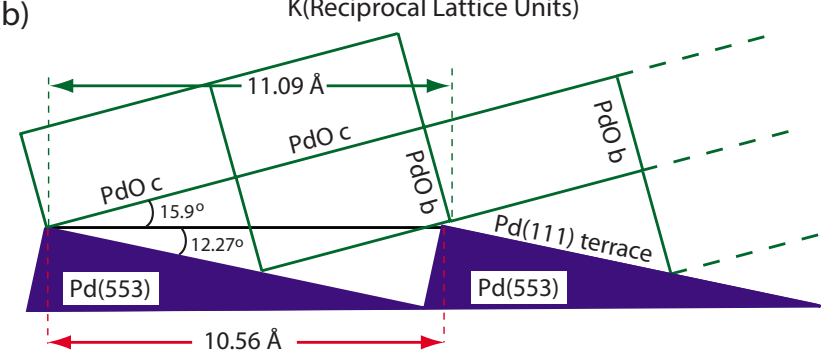

FIG. 10. (Color online) (a) Intensity distribution in the $H=0$ plane together with a schematic model of the PdO unit cell. (b) Illustration of the alignment of the $\mathrm{PdO}$ on the $\mathrm{Pd}(553)$ as deduced from (a).

$c$ axis of the $\mathrm{PdO}$ unit cell and the $\mathrm{PdO}(021)$ plane.

One possible orientation in which the $\mathrm{PdO}$ bulk oxide grows with respect to the $\operatorname{Pd}(553)$ surface is with the $a$ side along the steps and the (021) plane across the steps see Fig. 10 (b). There is a small mismatch between the step distance of the Pd(553) surface (10.56 $\AA$ ) and the length of the (021) plane in the $c$ direction (11.09 $\AA$ ). This difference could be compensated by adding one extra atom row to every fourth to fifth terrace, and if the macroscopic angle of the (553) surface is to be kept, larger (11 $\overline{1})$ planes have to be formed. The size of the (11) facets, as it is estimated from the width of the CTR, is roughly $25-30 \AA$. Thus, a very rough oxide is formed under these conditions.

\section{SUMMARY}

In summary, we have studied the oxidation of the $\operatorname{Pd}(553)$ surface. Our study shows that it is possible to investigate surface structures from UHV to atmospheric pressures by combining traditional electron based surface science techniques with SXRD and DFT. The UHV studies are crucial in order to understand observations at higher pressures using SXRD. At low oxygen pressures, the $\mathrm{O}$ atoms adsorb preferentially at the step edges, leaving the surface intact apart from surface relaxations. Increasing the $\mathrm{O}$ pressure above approximately $10^{-5}$ mbar results in the formation of a $\mathrm{PdO}(101)$-like surface oxide and a faceting into the (332) surface. Above 1 mbar, a rough PdO bulk oxide with (021) orientation is formed.

\section{ACKNOWLEDGMENTS}

This work was financially supported by the Swedish Research Council, the Crafoord Foundation, the Knut and Alice Wallenberg Foundation, the Austrian Fonds zur Förderung der wissenschaftlichen Forschung, and the European Union under Contract No. NMP3-CT-2003-505670 (NANO2). Support by the MAX-lab and ESRF staff is gratefully acknowledged.
${ }^{1}$ G. Ertl, H. Knözinger, and J. Weitkamp, Handbook of Heterogeneous Catalysis (Wiley, New York, 1997).

${ }^{2}$ M. D. Ackermann, T. M. Pedersen, B. L. M. Hendriksen, O. Robach, S. C. Bobaru, I. Popa, C. Quiros, H. Kim, B. Hammer, S. Ferrer, and J. W. M. Frenken, Phys. Rev. Lett. 95, 255505 (2005).

${ }^{3}$ B. L. M. Hendriksen and J. W. M. Frenken, Phys. Rev. Lett. 89, 046101 (2002).

${ }^{4}$ B. L. M. Hendriksen and J. W. M. Frenken, Surf. Sci. 552, 229 (2004).

${ }^{5}$ J. Gustafson, A. Mikkelsen, M. Borg, E. Lundgren, L. Köhler, G. Kresse, M. Schmid, P. Varga, J. Yuhara, X. Torrelles, C. Quiros, and J. N. Andersen, Phys. Rev. Lett. 92, 126102 (2004).

${ }^{6}$ Y. B. He, M. Knapp, E. Lundgren, and H. Over, J. Phys. Chem. B 109, 21825 (2005).

${ }^{7}$ A. Stierle, N. Kasper, H. Dosch, J. Gustafson, A. Mikkelsen, J. N. Andersen, and E. Lundgren, J. Chem. Phys. 122, 044706 (2005).

${ }^{8}$ G. Ketteler, D. F. Ogletree, H. Bluhm, H. J. Liu, E. L. D. Hebenstreit, and M. Salmeron, J. Am. Chem. Soc. 127, 18269 (2005).

${ }^{9}$ K. Reuter and M. Scheffler, Phys. Rev. B 65, 035406 (2001).

${ }^{10}$ N. Seriani, W. Pompe, and L. C. Ciacchi, J. Phys. Chem. B 110, 14860 (2006).
${ }^{11}$ E. Lundgren, J. Gustafson, A. Mikkelsen, J. N. Andersen, A. Stierle, H. Dosch, M. Todorova, J. Rogal, K. Reuter, and M. Scheffler, Phys. Rev. Lett. 92, 046101 (2004).

${ }^{12}$ H. Over, Y. D. Kim, A. P. Seitsonen, S. Wendt, E. Lundgren, M. Schmid, P. Varga, A. Morgante, and G. Ertl, Science 287, 1474 (2000).

${ }^{13}$ E. Lundgren, G. Kresse, C. Klein, M. Borg, J. N. Andersen, M. De Santis, Y. Gauthier, C. Konvicka, M. Schmid, and P. Varga, Phys. Rev. Lett. 88, 246103 (2002).

${ }^{14}$ M. Todorova, E. Lundgren, V. Blum, A. Mikkelsen, S. Gray, J. Gustafson, M. Borg, J. Rogal, K. Reuter, J. N. Andersen, and M. Scheffler, Surf. Sci. 541, 101 (2003).

${ }^{15}$ W. X. Li, L. Österlund, E. K. Vestergaard, R. T. Vang, J. Matthiesen, T. M. Pedersen, E. Lægsgaard, B. Hammer, and F. Besenbacher, Phys. Rev. Lett. 93, 146104 (2004).

${ }^{16} \mathrm{~J}$. Gustafson, A. Mikkelsen, M. Borg, J. N. Anderson, E. Lundgren, C. Klein, W. Hofer, M. Schmid, P. Varga, L. Köhler, G. Kresse, N. Kasper, A. Stierle, and H. Dosch, Phys. Rev. B 71, 115442 (2005).

${ }^{17}$ M. Schmid, A. Reicho, A. Stierle, I. Costina, J. Klikovits, P. Kostelnik, O. Dubay, G. Kresse, J. Gustafson, E. Lundgren, J. N. Anderson, H. Dosch, and P. Varga, Phys. Rev. Lett. 96, 146102 (2006). 
${ }^{18}$ J. Schnadt, A. Michaelides, J. Knudsen, R. T. Vang, K. Reuter, E. Lægsgaard, M. Scheffler, and F. Besenbacher, Phys. Rev. Lett. 96, 146101 (2006).

${ }^{19}$ E. Lundgren, A. Mikkelsen, J. N. Andersen, G. Kresse, M. Schmid, and P. Varga, J. Phys.: Condens. Matter 18, 481 (2006).

${ }^{20}$ P. Kostelnik, N. Seriani, G. Kresse, A. Mikkelsen, E. Lundgren, V. Blum, T. Sikola, P. Varga, and M. Schmid, Surf. Sci. 601, 1574 (2007).

${ }^{21}$ J. Rogal, K. Reuter, and M. Scheffler, Phys. Rev. Lett. 98, 046101 (2007).

${ }^{22}$ H. Over, A. P. Seitsonen, E. Lundgren, M. Schmid, and P. Varga, J. Am. Chem. Soc. 123, 11807 (2001).

${ }^{23}$ E. Lundgren, J. Gustafson, A. Resta, J. Weissenrieder, A. Mikkelsen, J. N. Andersen, L. Köhler, G. Kresse, J. Klikovits, A. Biederman, M. Schmid, and P. Varga, J. Electron Spectrosc. Relat. Phenom. 144, 367 (2005).

${ }^{24}$ G. Rupprechter, K. Hayek, and H. Hofmeistery, J. Catal. 173, 409 (1998).

${ }^{25}$ J. Assmann, D. Crihan, M. Knapp, E. Lundgren, E. Löffler, M. Muhler, V. Narkhede, H. Over, M. Schmid, A. Seitsonen, and P. Varga, Angew. Chem., Int. Ed. 44, 917 (2005).

${ }^{26}$ T. Schalow, M. Laurin, B. Brandt, S. Schauermann, S. Guimond, H. Kuhlenbeck, D. E. Starr, S. K. Shaikhutdinov, J. Libuda, and H. J. Freund, Angew. Chem., Int. Ed. 46, 7601 (2005).

${ }^{27}$ B. Lang, R. W. Joyner, and G. A. Somorjai, Surf. Sci. 30, 454 (1972).

${ }^{28}$ D. G. Castner and G. A. Somorjai, Surf. Sci. 83, 60 (1979).

${ }^{29}$ G. A. Somorjai, Chemistry in Two Dimensions: Surfaces (Cornell University Press, London, 1981).

${ }^{30}$ D. Hoogers and D. A. King, Surf. Sci. 286, 306 (1993).

${ }^{31}$ J. Gustafson, A. Resta, A. Mikkelsen, R. Westerström, J. N. Andersen, E. Lundgren, J. Weissenrieder, M. Schmid, P. Varga, N. Kasper, X. Torrelles, S. Ferrer, F. Mittendorfer, and G. Kresse, Phys. Rev. B 74, 035401 (2006).
${ }^{32}$ J. G. Wang, W. X. Li, M. Borg, J. Gustafson, A. Mikkelsen, T. M. Pedersen, E. Lundgren, J. Weissenrieder, J. Klikovits, M. Schmid, B. Hammer, and J. N. Andersen, Phys. Rev. Lett. 95, 256102 (2005).

${ }^{33}$ J. T. Yates, J. Vac. Sci. Technol. A 13, 1359 (1995).

${ }^{34}$ B. Hammer, O. H. Nielsen, and J. K. Nørskov, Catal. Lett. 46, 31 (1997).

${ }^{35}$ T. Zambelli, J. Wintterlin, J. Trost, and G. Ertl, Science 723, 1688 (1996).

${ }^{36}$ S. Dahl, A. Logadottir, R. C. Egeberg, J. H. Larsen, I. Chorkendorff, E. Törnqvist, and J. K. Nørskov, Phys. Rev. Lett. 83, 1814 (1999).

${ }^{37}$ R. Nyholm, J. N. Andersen, U. Johansson, B. N. Jensen, and I. Lindau, Nucl. Instrum. Methods Phys. Res. A 467-468, 520 (2001).

${ }^{38}$ A. Steinhäuser, N. Kasper, R. Weigel, and H. Dosch, Rev. Sci. Instrum. 75, 5302 (2004).

${ }^{39}$ S. Ferrer and F. Comin, Rev. Sci. Instrum. 66, 1674 (1995).

${ }^{40}$ P. Bernard, K. Peters, J. Alvarez, and S. Ferrer, Rev. Sci. Instrum. 70, 1478 (1999).

${ }^{41}$ G. Kresse and J. Hafner, Phys. Rev. B 47, 558 (1993).

${ }^{42}$ G. Kresse and J. Furthmuller, Comput. Mater. Sci. 6, 15 (1996).

${ }^{43}$ P. E. Blöchl, Phys. Rev. B 50, 17953 (1994).

${ }^{44}$ G. Kresse and D. Joubert, Phys. Rev. B 59, 1758 (1999).

${ }^{45}$ J. P. Perdew, J. A. Chevary, S. H. Vosko, K. A. Jackson, M. R. Pederson, D. J. Singh, and C. Fiolhais, Phys. Rev. B 46, 6671 (1992).

${ }^{46}$ A. Stierle, N. Kasper, H. Dosch, E. Lundgren, J. Gustafson, A. Mikkelsen, and J. N. Andersen, J. Chem. Phys. 122, 044706 (2005).

${ }^{47}$ K. Yagi and H. Fukutani, Surf. Sci. 412, 489 (1998).

${ }^{48}$ J. N. Andersen, D. Hennig, E. Lundgren, M. Methfessel, R. Nyholm, and M. Scheffler, Phys. Rev. B 50, 17525 (1994). 